\title{
Photoluminescence of Charged Excitons in Fractional Quantum Hall Systems
}

\author{
A. Wóss \\ Institute of Physics, Wrocław University of Technology \\ Wybrzeże Wyspiańskiego 27, 50-370 Wrocław, Poland
}

\begin{abstract}
Neutral and charged excitonic complexes formed in integral and fractional quantum Hall systems are discussed. They are bound states of a small number of charged quasiparticles (e.g., conduction electrons and valence holes, reversed-spin electrons and spin holes, Laughlin quasielectrons and quasiholes, composite fermions) that occur in an electron system under specific conditions (electron density, well width, electric and magnetic fields, etc.). The examples are interband neutral and charged excitons, "anyon excitons", spin waves, skyrmions, and "skyrmion excitons". Their possible decay processes include radiative recombination, experimentally observed in photoluminescence or far infrared emission, or spin transitions, important in the context of nuclear spin relaxation.
\end{abstract}

PACS numbers: 71.35.Ji, 71.35.Ee, 71.35.Pq

\section{Introduction}

The two-dimensional electron gas (2DEG) in a high magnetic field $B$ and at low temperature $T$ has a number of remarkable properties, resulting from interplay of single-particle Landau level (LL) quantization (of either electrons or other charged excitations relevant under particular conditions) and many-body interaction within degenerate LL's. The arguably most prominent macroscopic phenomena associated with the fascinating physics of the 2DEG at high $B$ and low $T$ are integral $[1,2]$ and fractional $[3,4]$ quantum Hall effects observed in transport experiments. Some of the most fundamental concepts developed to understand these phenomena are the fractionally charged Laughlin quasiparticles (QP's) [4-6] and composite fermions (CF's) [7]. However, also the optical and spin properties of the 2DEG have been intensively studied in this regime, both experimentally [8-10] and theoretically [11-15]. 
One of the most important microscopic effects at high $B$ is that any charged particle moves along a closed (cyclotron) orbit, rather than an open straight line. As a consequence, particles of charge $q$ can be confined to a finite area scaled by the magnetic length $\lambda=\sqrt{h c / q B}$ within the lowest $L L$ (i.e., at no additional kinetic energy cost). This is in striking contrast to the situation at $B=0$, where the corresponding confinement energy coincides with the LL ground state energy $\frac{1}{2} \hbar \omega_{c}$. The ability of charged particles to occur in a "compact" form (similar to classical charged particles of finite size) stands behind many features characteristic of a 2DEG in high $B$, from the simplest ones, such as degeneracy of the ground state, to those understood only more recently, such as essential role of two-dimensionality, Wigner crystallization, or Laughlin correlations.

The excitonic complexes in 2DEG, being a central theme of this paper, are also formed because of the finite size of Landau orbitals. Similarly as happens for classical charges, one can always expect that positive and negative particles in a degenerate LL will arrange themselves into neutral or charged excitonic bound states, depending on the relative numbers of the two species. The excitonic complexes, generally consisting of a small number of relevant elementary charged excitations (positively and negatively charged QP's of various type depending on a particular form of the $e-e$ correlations in the underlying 2DEG), can often be considered as nearly free particles with well-defined single-particle properties. These properties (charge, size, longitudinal/angular momentum, spin, binding energy, or oscillator strength for a particular decay, etc.) determine the response of the 2DEG to the experimental perturbation. In particular, being weakly coupled to one another or to the electrons, excitonic complexes recombine obeying simple selection rules that result from their geometric (2D translational) or dynamical (particle-hole) symmetries. These simple symmetries often persist under experimental conditions despite complicated $e-e$ correlations or such typical symmetry-breaking mechanisms as disorder or collisions, and they greatly simplify the response of the system.

Sometimes, such simplification is even undesirable as it can make the experiment only sensitive to the simple properties of the excitonic complexes, and quite insensitive to some of the specific properties of the underlying 2DEG. For example, it has long been predicted that the photoluminescence (PL) spectrum at $B=\infty$ contains no information about the $e-e$ correlations (e.g., the presence or charge of Laughlin QP's in the fractional quantum Hall regime) regardless of disorder [14]. Instead, the PL spectrum is reduced to a single transition corresponding to the recombination of the $k=0$ ground state of a neutral exciton, and either decreasing $B$ in order to allow Landau level mixing or applying an electric field to separate electron and hole layers is needed for PL to probe $e-e$ interactions.

Another example is related to a prediction $[16,17]$ that the most strongly bound complex involving conduction electrons and a valence hole $(v)$ in very high $B$ is the triplet state of a charged exciton $\left(X^{-}=2 e+v\right)$. This state is nonradiative because of both geometrical and dynamical symmetry, and has not been 
experimentally confirmed in earlier experiments in high $B$ [18], but only quite recently $[19,20]$, when special measures were taken to detect its weak PL signal. While breaking the dynamical symmetry at $B<\infty$ is by no means surprising, the fact that collisions of an $X^{-}$with surrounding electrons do not relax the geometrical selection rule associated with the angular momentum conservation is a nice demonstration of Laughlin correlations of an $X^{-}$with other negative charges [21]. As a result of these correlations, at least at small values of the filling factor $\nu$, the $X^{--}$'s remain spatially isolated and avoid high energy collisions with one other or with electrons to become true QP's of a 2DEG containing additional valence holes [22].

This article is a review of similarities and differences between various excitonic complexes formed in quantum Hall systems: interband excitons, anyon excitons, skyrmions, and skyrmion excitons. Some of the results have been earlier published in Refs. [21-26], but Figs. 1b, 2, 3, and 8 are presented here for the first time.

\section{Model}

The numerical results are obtained by numerical diagonalization of the interaction Hamiltonian of a finite number $N$ of electrons (and, sometimes, one or more valence holes) confined on a spherical surface of radius $R$. In this model, the radial magnetic field $B$ is due to a monopole in the center [5]. The monopole strength $2 Q$ is defined in units of elementary flux $\phi_{0}=h c / e$, so that $4 \pi R^{2} B=2 Q \phi_{0}$ and the electron magnetic length is $\lambda=R / \sqrt{Q}$. The single-particle states ("monopole harmonics") are the eigenstates of angular momentum $l$ and its projection $m$. The energies $\varepsilon$ fall into $(2 l+1)$-fold degenerate angular momentum shells separated by the cyclotron energy $\hbar \omega_{c}$. The $n$-th $(n \geq 0)$ shell (LL) has $l=Q+n$ and thus $2 Q$ is a measure of the system size through the LL degeneracy. Due to the spin degeneracy, each $l$-shell is further split by the Zeeman gap, $E_{\mathrm{Z}}$.

Using a composite index $i=[n m \sigma]$ ( $\sigma$ is the spin projection), the Hamiltonian of interacting particles can be written as $H=\sum c_{i \alpha}^{\dagger} c_{i \alpha} \varepsilon_{i \alpha}+$ $\sum c_{i \alpha}^{\dagger} c_{j \beta}^{\dagger} c_{k \beta} c_{l \alpha} V_{i j k l \alpha \beta}$, where $c_{i \alpha}^{\dagger}$ and $c_{i \alpha}$ create and annihilate particle $\alpha$ (conduction electron $e$ or valence hole $v$, reversed-spin electron $e_{\mathrm{R}}$ or spin hole $h$, etc.) in state $i$ with energy $\varepsilon_{i \alpha}$, and $V_{i j k l \alpha \beta}$ are the interaction (Coulomb) matrix elements. Hamiltonian $H$ is diagonalized in the basis of Slater determinants. The result is the set of many-body eigenenergies and eigenvectors. The energies $E$ will be shown as a function of the conserved orbital $\left(L\right.$ and $\left.L_{z}\right)$ and spin $(S$ and $S_{z}$ ) quantum numbers. To interpret the results obtained in the spherical geometry for the infinite planar system, $L$ and $L_{z}$ must be appropriately translated into the corresponding planar quantities [22, 25]. For example, for the (charge or spin) wave eigenstates that carry no net charge, angular momentum $L$ must be replaced by wave vector $k=L / R$, while for the eigenstates corresponding 
to charged excitations $L$ and $L_{z}$ are connected with planar angular momentum projection $\mathcal{M}$ and its center-of-mass component $\mathcal{M}_{\mathrm{CM}}$. The eigenvectors $|\psi\rangle$ are needed to calculate spectral functions to describe PL or other decay processes, $\tau_{i f}^{-1}=|\langle f|\mathcal{P}| i\rangle|^{2}$, where $\psi=i$ or $f$ are the initial and final states, respectively, and $\mathcal{P}$ is the appropriate transition operator.

\section{Neutral and charged interband excitons}

The energy spectra of an $X^{-}=2 e+v$ in a symmetric GaAs quantum well of width $w=11.5 \mathrm{~nm}$ were calculated in Ref. [22] taking into account the effects of LL mixing, finite well width, anisotropy and $w$ - and $B$-dependence of the hole mass, and the realistic Zeeman gap $E_{\mathrm{Z}}$. From these spectra one can calculate the $X^{-}$binding energy, defined as $\Delta=E_{X}-E$, where $E_{X}$ is the exciton energy.

Because the emission of a photon does not change angular momentum of the (envelope) wave function, and because the electron left in the lowest LL after the $X^{-}$recombination has $l=Q$, only those $X^{-}$states at $L=Q$ are optically active. Of all bound $X^{-}$states, three are of particular importance. The $X_{\mathrm{s}}^{-}$(singlet) and $X_{\mathrm{tb}}^{-}$(triplet-bright) are the only strongly bound radiative states, while $X_{\mathrm{td}}^{-}$ (triplet-dark) has the lowest energy of all non-radiative states. The relative energy of different $X^{-}$states depends on experimentally variable parameters (e.g., $B, w$, or $E_{\mathrm{Z}}$ ), and indeed, the transition between the $X_{\mathrm{s}}^{-}$and $X_{\mathrm{td}}^{-}$states has been found in the spectra [22]. The binding energies $\Delta$ of the three $X^{-}$states, extrapolated to the $R / \lambda=\sqrt{Q} \rightarrow \infty$ limit, are plotted in Fig. 1 as a function of $B$. Frame (a) is for a symmetric $w=11.5 \mathrm{~nm}$ well in which the $X_{\mathrm{s}}^{-} \leftrightarrow X_{\mathrm{td}}^{-}$transition is found at $B \approx 30 \mathrm{~T}$. We also find that the $X_{\mathrm{tb}}^{-}$state is about two times "brighter" than $X_{\mathrm{s}}^{-}$in the whole range of $B$. In realistic wells with significant electron density $\varrho$, the electric field produced by the doping layer splits $e$ and $v$ wave functions in the $z$-direction. As a result, the $X_{\mathrm{s}}^{-} \leftrightarrow X_{\mathrm{td}}^{-}$transition moves toward lower $B$. In frame (b) we plot the binding energies for a high density $\varrho=2.5 \times 10^{11} \mathrm{~cm}^{-2}$. Clearly, all $X^{-}$states unbind at $B>20 \mathrm{~T}$, except for the $X_{\mathrm{td}}^{-}$with $\Delta_{\mathrm{td}} \sim 0.7 \mathrm{meV}$.

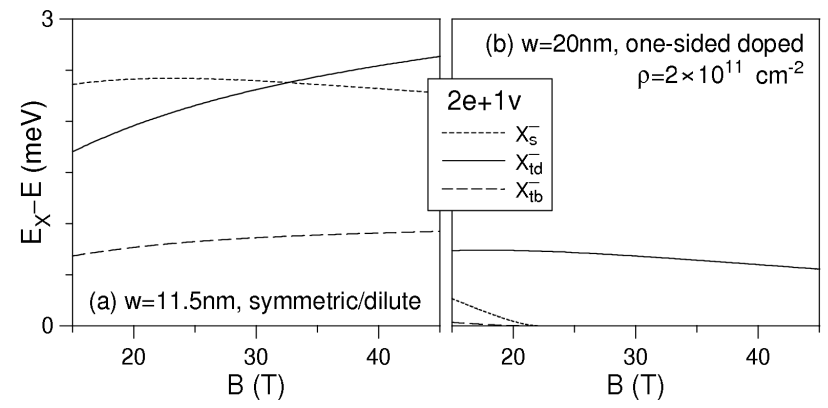

Fig. 1. Binding energies $\Delta=E_{X}-E$ of different $X^{-}$states in a symmetric (a) and strongly asymmetric (b) GaAs quantum well as a function of magnetic field $B$. 
An interesting experimental result is a weak dependence of the $X^{-}$binding energy spectrum on the well width $w$. In Ref. [27], data obtained in a few different samples was shown to fall nicely on the same three curves $\Delta(B)$. Numerically, it looks different, as especially $\Delta_{\mathrm{s}}$ is quite sensitive to $w$. The width dependences $\Delta(w)$ calculated for $B=20 \mathrm{~T}$ are shown in Fig. 2a. One effect that was neglected in the calculation of results in Fig. 1 is the subband mixing, i.e. dependence of the $e$ and $v$ wave functions in the $z$-direction on the interactions. To estimate the effect on the $X^{-}$energy spectrum we combined exact diagonalization in the $x y$ plane with variational calculation along the $z$-axis - by varying effective layer widths $w_{\mathrm{e}}^{*}$ and $w_{\mathrm{h}}^{*}$ and repeating the $x y$-diagonalization for each pair. For $w^{*} / w<1$ the interactions are stronger while the kinetic energy is larger, and an optimum combination of $w_{\mathrm{e}}^{*}$ and $w_{\mathrm{h}}^{*}$ describes each of the $X$ or $X^{-}$ground states.

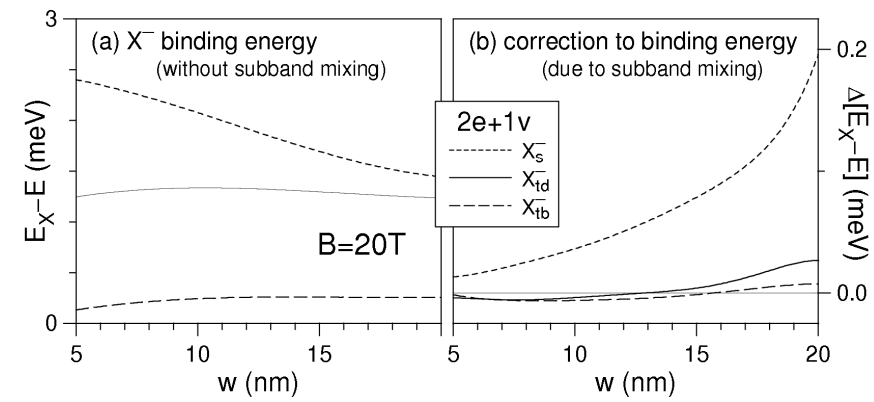

Fig. 2. (a) Binding energies $\Delta$ (neglecting subband mixing) of different $X^{-}$states in a symmetric GaAs quantum well as a function of width $w$, in magnetic field $B=20 \mathrm{~T}$.

(b) Correction to the binding energy due to subband mixing.

The correction to the binding energy at $B=20 \mathrm{~T}$ is shown in Fig. $2 \mathrm{~b}$. For $X_{\mathrm{s}}^{-}$ it partially compensates for the $w$-dependence in Fig. 2a. It seems plausible that a larger correction (and weaker overall $w$-dependence) would result if correlations in the $z$-direction were also included. While in narrow wells $(w \sim 10 \mathrm{~nm})$ and in moderate fields $(B \sim 20 \mathrm{~T})$ the correction is as small as $\sim 2 \%$ (and the subband mixing can be neglected), it can reach $50 \%$ in wide wells $(\sim 20 \mathrm{~nm})$ and in the strongest fields $(\sim 50 \mathrm{~T})$, indicating that subband mixing must be fully (including correlations) taken into account in these systems.

The actual reduction of layer widths measured by $\xi=w^{*} / w$ is shown in Fig. 3 as a function of both $B$ (for $w=12 \mathrm{~nm}$ ) and $w$ (for $B=20 \mathrm{~T}$ ). Let us begin with a nearly obvious observation that the effect depends not independently on $B$ and $w$, but (roughly) on the ratio $w / \lambda \propto w \sqrt{B}$. Clearly, the reduction is larger for the (heavier) hole than for the (lighter) electron. Also, it strongly depends on the particular $X$ or $X^{-}$bound state, being the strongest for the hole in the $X_{\mathrm{s}}^{-}$ state. Interestingly, the asymmetry between $\xi_{\mathrm{e}}$ and $\xi_{\mathrm{h}}$ is much larger for $X^{-}$states having two electrons than for $X$ with only one $\left(w_{\mathrm{e}}{ }^{*} \approx w_{\mathrm{e}}\right.$ for $X^{-}$'s). The values 


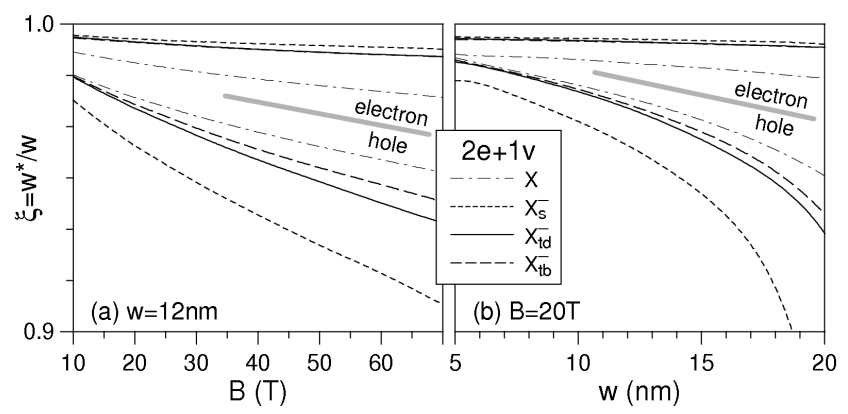

Fig. 3. Normalized effective layer widths $\xi=w^{*} / w$ of electrons and holes in different $X$ and $X^{-}$states in a symmetric GaAs quantum well, as a function of magnetic field $B$ (a) and width $w(\mathrm{~b})$, for $w=12 \mathrm{~nm}$ and $B=20 \mathrm{~T}$, respectively.

of $\xi$ for the hole in $X_{\mathrm{s}}^{-}$range from under $5 \%$ in narrow wells and at moderate $B$ (to justify the neglection of the effect) to as large as $\sim 50 \%$ for $w \sim 20 \mathrm{~nm}$ and $B \sim 50 \mathrm{~T}$ (to completely invalidate the lowest subband approximation).

Even in dilute systems, one might expect that collisions with surrounding electrons can affect the $X^{-}$recombination and, e.g., allow for weak emission from $X_{\mathrm{td}}^{-}$. The surprising experimental fact that the effect of such collisions is minimal [18-20] results from Laughlin $e-X^{-}$correlations [21]. The $3 e+v$ energy spectra, in which the lowest bands describe repulsion of different $e-X^{-}$pairs, were calculated in Ref. [22]. The dependence of pair interaction energy $V$ on pair angular momentum $L$ is the pseudopotential, which completely determines correlations in a degenerate LL. Because the $e-X^{-}$pseudopotential $V(L)$ is superharmonic [28], Laughlin correlations result. They mean that the $e-X^{-}$pair states with the largest Coulomb energy are avoided (high-energy $e-X^{-}$collisions do not occur).

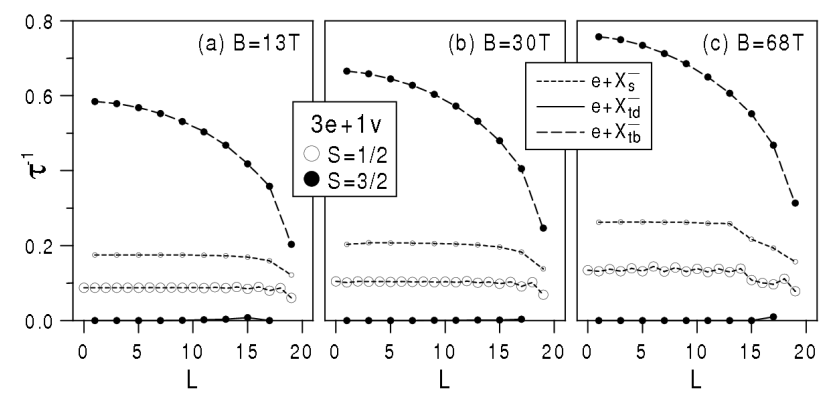

Fig. 4. Oscillator strengths $\tau^{-1}$ of different $X^{-}$states interacting with an electron in a symmetric GaAs quantum well of width $w=11.5 \mathrm{~nm}$ at a magnetic field $B=13 \mathrm{~T}$ (a), $30 \mathrm{~T}$ (b), and $68 \mathrm{~T}$ (c), calculated on Haldane sphere with LL degeneracy $2 Q+1=21$, and plotted as a function of the $e-X^{-}$pair angular momentum $L$. 


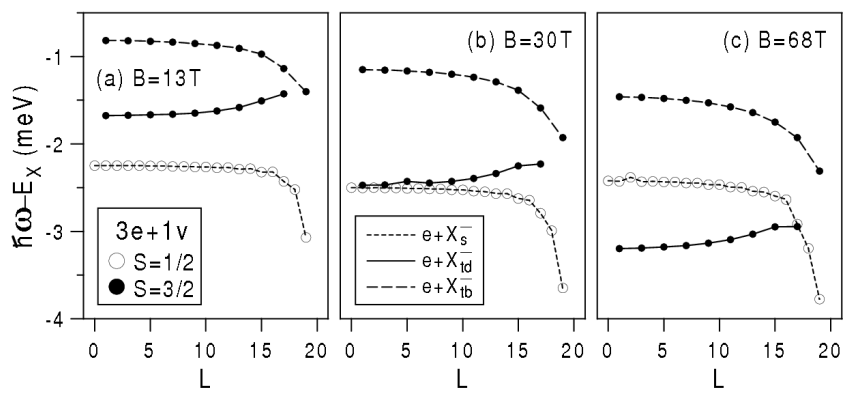

Fig. 5. The same as in Fig. 4 but with recombination energy $\hbar \omega$ shown on vertical axes.

In Figs. 4 and 5 we plot the oscillator strengths $\tau^{-1}$ and emission energies $\hbar \omega$ for the $3 e+v$ eigenstates corresponding to an $X^{-}$interacting with an electron. In both figures, the horizontal axes give pair angular momentum $L$ which in a Laughlin correlated system is simply related to the LL filling factor $\nu$ (only the $L \leq l_{X^{-}}+l_{\mathrm{e}}-\mu$ pair states occur at $\nu \leq \mu^{-1}$ ). As expected, for small $L$ (i.e., very dilute $2 \mathrm{DEG}$ ) both $\hbar \omega$ and $\tau^{-1}$ converge to the values appropriate for single $X^{-}$'s plotted in Fig. 1, meaning that there is no significant effect of the $e-X^{-}$interactions on the $X^{-}$recombination at small $\nu$. Somewhat surprisingly, the Laughlin correlations prevent considerable increase in $\tau_{\mathrm{td}}^{-1}$ through interaction with electrons even at $\nu \approx \frac{1}{3}$. This justifies a simple picture of PL in a dilute 2DEG, according to which emission occurs from isolated, well-defined bound complexes ( $X$ and $X^{-}$'s), and it is virtually insensitive to $\nu$. This explains the difficulty in detecting the $X_{\mathrm{td}}^{-}$peak $[19,20]$ even in the PL spectra [18] showing a strong recombination of a higher-energy triplet state $X_{\mathrm{tb}}^{-}$. An interesting feature in Fig. 5 is merging of $\hbar \omega_{\mathrm{t}} \mathrm{b}$ and $\hbar \omega_{\mathrm{td}}$ which also was observed experimentally at $\nu \approx \frac{1}{3}$ [19].

\section{Anyon excitons}

The fractionally charged "anyon excitons" are expected to form in asymmetric quantum wells, in which the perpendicular electric field spatially separates electron $(e)$ and valence hole $(v)$ layers by a distance $d \sim \lambda[23,29]$. In such situation, the strength and resolution of the $v-e$ attraction become smaller than the characteristic 2DEG energy and length scales, and the 2DEG retains its original Laughlin correlations even in the presence of an optically injected hole $v$. Instead of being screened by "real" electrons $e$, the charge of $v$ is screened by fractionally charged quasielectrons (QE's) [23] or reversed-spin quasielectrons $\left(\mathrm{QE}_{\mathrm{R}}\right)[6,30]$.

A few examples of the energy spectra of $9 e+v$ systems at the values of $2 Q$ corresponding to different QE numbers $N_{\mathrm{QE}}$ in the Laughlin $\nu=\frac{1}{3}$ state of 9 electrons interacting with the hole are shown in Figs. 6 and 7 for $d=0$ and $2 \lambda$. They were calculated in Ref. [23], without taking into account the LL mixing or finite well width, so $d / \lambda$ is an effective parameter controlling the strength and 


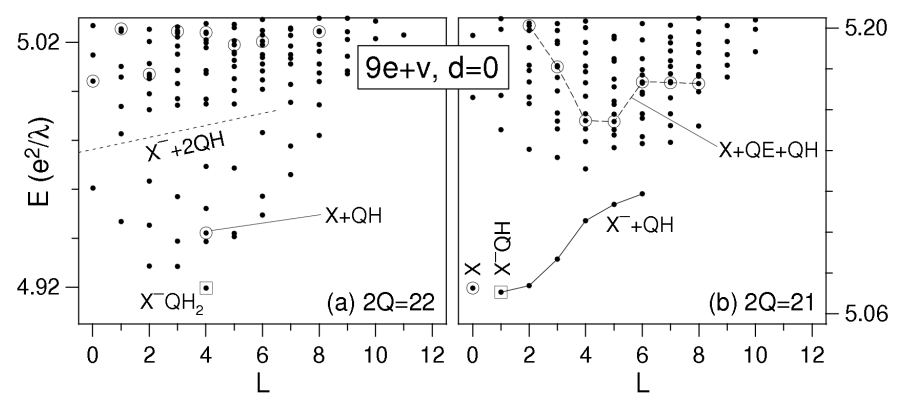

Fig. 6. Energy spectra (energy $E$ vs. angular momentum $L$ ) of an ideal $9 e+v$ system (no LL mixing and zero width) calculated on Haldane sphere with LL degeneracy $2 Q+1=22$ (a) and 21 (b). The $e-v$ layer separation is $d=0$.

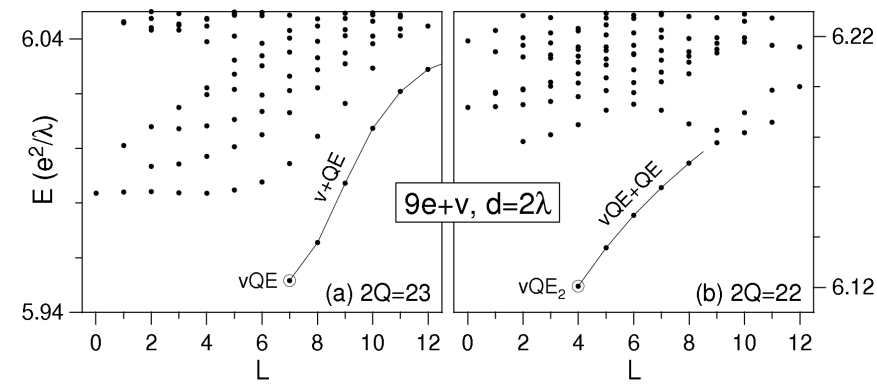

Fig. 7. The same as in Fig. 6 but for $d=2 \lambda$ and different values of $2 Q$.

resolution of the hole perturbation potential, rather than an actual displacement of $e$ and $v$ wave functions.

In Fig. $6(d=0)$, the $X_{\mathrm{td}}^{-}$(the only bound $X^{-}$state in the lowest LL), is the most stable QP, and the anyon excitons do not form. The open circles mark the so-called "multiplicative" states in which the $L=0$ exciton decouples from the remaining 8 electrons due to the "hidden" symmetry [13]. All other low-energy $9 e+v$ states contain an $X^{-}$interacting with the remaining 7 electrons. These states are well described by a CF model generalized [21] for the two-component $\left(7 e+X^{-}\right)$Laughlin liquid. At $2 Q \geq 21$, the lowest-energy $7 e+X^{-}$states contain additional Laughlin quasiholes (QH's). The residual $\mathrm{QH}-X^{-}$attraction, whose pseudopotential can be extracted from the $X^{-}+\mathrm{QH}$ band in frame (b), leads to the formation of $X^{-} \mathrm{QH}$ and $X^{-} \mathrm{QH}_{2}$ weakly bound states identified in frames (a) and (b). These states may have been detected in a recent experiment [31].

In Fig. $7(d=2 \lambda)$, new low-energy bands of "anyon exciton" states occur. The isolated $v \mathrm{QE}, v \mathrm{QE}_{2}$, and $v \mathrm{QE}_{3}$ states are the ground states in the spectra corresponding to $N_{\mathrm{QE}}=1,2$, and 3 , respectively. Their angular momenta $l_{\mathrm{AX}}$ are obtained by adding $l_{h}=Q$ and $l_{\mathrm{QE}}=Q^{*}+1$, where $2 Q^{*}=$ $2 Q-2(N-1)$ is the effective monopole strength in the CF picture $[7,28]$ and $2 Q=$ 
$3(N-1)-N_{\mathrm{QE}}$. Similarly, the angular momenta of states containing an anyon exciton and the excess QP's result from adding $l_{\mathrm{AX}}$ and $l_{\mathrm{QP}}$. In analogous spectra for unpolarized electrons, the "reversed-spin anyon excitons" $v \mathrm{QE}_{\mathrm{R}}, v \mathrm{QE}_{\mathrm{R}} \mathrm{QE}$, and $v\left(\mathrm{QE}_{\mathrm{R}}\right)_{2}$ additionally appear, in which one or more $\mathrm{QE}$ 's are replaced by $\mathrm{QE}_{\mathrm{R}}$ 's.

Can the $e-v$ layer separation sufficient for the formation of anyon excitons be realized in realistic samples? In Fig. $8 \mathrm{a}$ we plot the squared overlaps $\zeta$ (in the $x y$ plane) between the exact $v \mathrm{QE}_{n}$ wave functions and the actual lowest energy eigenstates (at the same values of $L$ ) calculated in the $8 e+v$ system for the varied well width $w$ and assuming one-sided doping and electron density $\varrho=$ $2.5 \times 10^{11} \mathrm{~cm}^{-2}$. Clearly, anyon excitons are expected to occur in wells with $w>40 \mathrm{~nm}$ at this density. Separation of $e-v$ layers needed to break up the $X^{-}$ at the same time reduces oscillator strengths of anyon excitons. To estimate this effect, in Fig. $8 \mathrm{~b}$ we show the squared $v-e$ overlap in the $z$-direction, that scales all total $v \mathrm{QE}_{n}$ oscillator strengths.

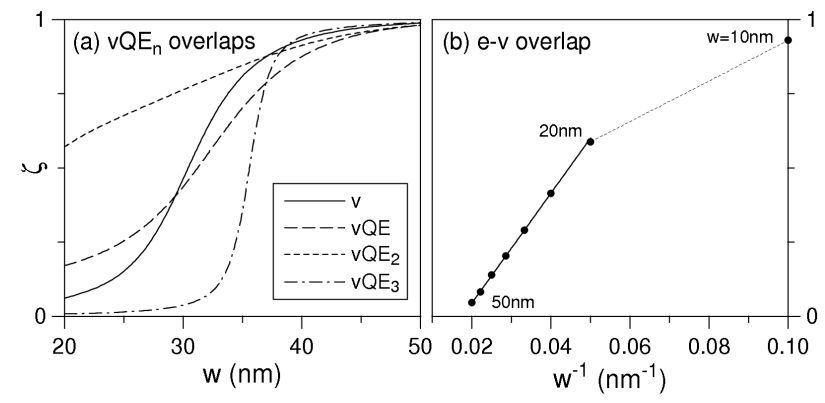

Fig. 8. (a) Squared overlaps $\zeta$ between $v \mathrm{QE}_{n}$ wave functions and the actual lowest-energy states at the same angular momentum $L$ calculated as a function of well width $w$ for a constant electron density $\varrho=2.5 \times 10^{11} \mathrm{~cm}^{-2}$. (b) Squared electron-hole overlap in the $z$-direction calculated as a function of inverse well width $w^{-1}$ for the same $\varrho=2.5 \times 10^{11} \mathrm{~cm}^{-2}$. Roughly linear dependence between $w=20$ and $50 \mathrm{~nm}$ is marked with a solid line; thin dashed line only connects the points for $w=10$ and $20 \mathrm{~nm}$.

Similarly as it was for $X^{-}$'s, the translational symmetry of an isolated anyon exciton leads to the conservation of its $L$ and $L_{z}$ in the emission process. This leads to strict optical selection rules that can only be broken by collisions or disorder. The recombination of an anyon exciton state formed in a Laughlin liquid occurs through annihilation of a well-defined number of QE's and creation of an appropriate number of QH's [29]. It turns out that the processes involving more than the minimum number of QP's all have negligible intensity, which for $\nu=\frac{1}{3}$ leaves only the following four possible recombination events: $v+n \mathrm{QE} \rightarrow$ $(3-n) \mathrm{QH}+\gamma$, where $n=0,1,2$, or 3 , and $\gamma$ denotes the photon. When the angular momentum conservation law is applied to the above recombination 
events, we obtain [24] that the only radiative anyon excitons are $v \mathrm{QE}^{*}$ (the first excited state of a $v-\mathrm{QE}$ pair), $v \mathrm{QE}_{\mathrm{R}}$, and $v \mathrm{QE}_{2}$, while all others (including $v \mathrm{QE}$ ) are "dark." Because the formation of radiative anyon excitons depends on the presence of $\mathrm{QE}$ 's or $\mathrm{QE}_{\mathrm{R}}$ 's in the 2DEG, the magneto-PL spectrum must change discontinuously at $\nu=\frac{1}{3}[8]$.

\section{Spin waves and skyrmions}

The integral quantum Hall system near $\nu=1$ with spin excitations can contain a small number of reversed-spin electrons $e_{\mathrm{R}}$ and spin holes $h$, which makes it very similar to the dilute system of conduction electrons $e$ and valence holes $v$. The important difference is that the energy of a $k=0$ spin wave (i.e., a $e_{\mathrm{R}}-h$ pair, playing the role of an interband exciton $X$ ) is equal to the electron Zeeman splitting, $E_{\mathrm{Z}}$, which can be made small compared to the interaction energy, $e^{2} / \lambda$. Therefore, it is possible to achieve experimentally a situation in which the skyrmions $S_{K}^{+}=h+K \times h e_{\mathrm{R}}$ or antiskyrmions $S_{K}^{-}=K e_{\mathrm{R}}+K \times h e_{\mathrm{R}}$ (the $S_{1}^{-}=2 e_{\mathrm{R}}+h$ being the analogue of an interband dark triplet $X^{-}$) are stable ground states of the system [15, 17, 25], with infinite lifetimes, not limited by radiative recombination.

The $e_{\mathrm{R}}-h$ annihilation process analogous to the $e-v$ radiative emission occurs by hyperfine coupling to localized nuclear spins. However, the selection rule for such a process is completely different from that governing PL. The appropriate spectral function $\tau^{-1}(k)$ for the spin wave creation/destruction has a maximum at $k \lambda \sim 1$, corresponding to the characteristic size of the electron cyclotron orbit [32]. As a consequence, a $S^{-}$can decay via spin-flip (in contrast to the dark nature of its $X^{-}$interband analog). Actually, processes of this type $\left(S_{K} \leftrightarrow S_{K \pm 1}\right)$ are largely responsible for the nuclear spin relaxation in quantum Hall systems).

Spin waves and skyrmions also occur in the fractional quantum Hall regime (near $\nu=\frac{1}{3}$ ), only with $\mathrm{QE}_{\mathrm{R}}$ and $\mathrm{QH}$ replacing $e_{\mathrm{R}}$ and $h$. In the $\mathrm{CF}$ picture, they appear identical to those at $\nu=1$, only with CF's playing the role of the electrons.

\section{Skyrmion excitons}

When a valence hole $v$ is introduced into a quantum Hall system with a small $E_{\mathrm{Z}}$, it may substitute for one of the spin holes $h$ in a skyrmion or antiskyrmion to form a skyrmion exciton [26, 33]. Such a complex may decay both radiatively and via hyperfine interaction to nuclear spins. It also has a richer energy spectrum as the two kinds of holes, $h$ and $v$ become typically distinguishable. Unlike in a dilute $e-v$ system, where also three kinds of particles ( $e$ could have two different spins) were involved in a $X_{\mathrm{s}}^{-}$state, different orbitals of $h$ and $v$ holes (e.g., due to different effective masses or different response to the electric field) make the $e-h$ and $e-v$ interactions different. This prevents the mapping of a $h-v-e_{\mathrm{R}}$ 
system on a simple two-(iso)spin $e \uparrow-e \downarrow-v$ system with (iso)spin-symmetric interactions.

When a $v$ is added to a quantum Hall state at $\nu \leq 1$, there are no negatively charged excitations it could bind. But if $E_{\mathrm{Z}}$ is sufficiently small, $v$ may induce and bind one or more spin waves to form a skyrmion exciton, $v \rightarrow v h e \rightarrow v(h e)_{2} \rightarrow \ldots$. The situation is more complicated in the presence of reversed-spin electrons or skyrmions (at $\nu>1$ ). Being negatively charged, they are attracted to $v$ and, depending on $E_{\mathrm{Z}}$ or $d$, they can bind to it to form neutral or charged $h-v-e_{\mathrm{R}}$ states. Our calculations in ideal systems indicate that the binding energy for the $v e_{\mathrm{R}}+h e_{\mathrm{R}} \rightarrow v h\left(e_{\mathrm{R}}\right)_{2}$ process is negative for $d \leq 1.35 \lambda$. This means that (in symmetric structures) attraction between $v$ and $S_{1}^{-}=h\left(e_{\mathrm{R}}\right)_{2}$ (or a larger skyrmion) causes breakup of the latter and emission of free spin waves: $v+e_{\mathrm{R}}\left(h e_{\mathrm{R}}\right)_{K} \rightarrow$ $v e_{\mathrm{R}}+K \times h e_{\mathrm{R}}$. This would make the equilibrium PL signal come from the same initial state, $v e_{\mathrm{R}}$, regardless of the size $K$ of the skyrmions present in the 2DEG. On the other hand, the $v e_{\mathrm{R}}$ exciton might attract a second $e_{\mathrm{R}}$ or $S^{-}$to acquire charge and become able to induce and bind one or more spin waves.

\section{Acknowledgment}

The author thanks J.J. Quinn, P. Hawrylak, M. Potemski, L. Bryja, I. Bar-Joseph, M. Bayer, S. Crooker, and C. Schüller for helpful discussions, and acknowledges support from grants 2P03B02424 and PBZ-Min-008/P03/03 of Polish Ministry of Scientific Research and Information Technology.

\section{References}

[1] K. von Klitzing, G. Dorda, M. Pepper, Phys. Rev. Lett. 45, 494 (1980).

[2] R. Laughlin, Phys. Rev. B 23, 5632 (1981).

[3] D.C. Tsui, H.L. Störmer, A.C. Gossard, Phys. Rev. Lett. 48, 1559 (1982).

[4] R. Laughlin, Phys. Rev. Lett. 50, 1395 (1983).

[5] F.D.M. Haldane, Phys. Rev. Lett. 51, 605 (1983).

[6] E. Rezayi, Phys. Rev. B 36, 5454 (1987).

[7] J.K. Jain, Phys. Rev. Lett. 63, 199 (1989).

[8] D. Heiman, B.B. Goldberg, A. Pinczuk, C.W. Tu, A.C. Gossard, J.H. English, Phys. Rev. Lett. 61, 605 (1988).

[9] K. Kheng, R.T. Cox, Y. Merle d'Aubigné, F. Bassani, K. Saminadayar, S. Tatarenko, Phys. Rev. Lett. 71, 1752 (1993).

[10] R. Tycko, S.E. Barrett, G. Dabbagh, L.N. Pfeiffer, K.W. West, Science 268, 1460 (1995).

[11] C. Kallin, B.I. Halperin, Phys. Rev. B 30, 5655 (1984).

[12] I.D. Vagner, T. Maniv, Phys. Rev. Lett. 61, 1400 (1988). 
[13] A. MacDonald, E. Rezayi, Phys. Rev. B 42, 3224 (1990).

[14] A. MacDonald, E. Rezayi, D. Keller, Phys. Rev. Lett. 68, 1939 (1992).

[15] S.L. Sondhi, A. Karlhede, S.A. Kivelson, E.H. Rezayi, Phys. Rev. B 47, 16419 (1993).

[16] A. Wójs, P. Hawrylak, Phys. Rev. B 51, 10880 (1995).

[17] J.J. Palacios, D. Yoshioka, A. MacDonald, Phys. Rev. B 54, 2296 (1996).

[18] M. Hayne, C.L. Jones, R. Bogaerts, C. Riva, A. Usher, F.M. Peeters, F. Herlach, V.V. Moshchalkov, M. Henini, Phys. Rev. B 59, 2927 (1999).

[19] G. Yusa, H. Shtrikman, I. Bar-Joseph, Phys. Rev. Lett. 87, 216402 (2001).

[20] C. Schüller, K.-B. Broocks, Ch. Heyn, D. Heitmann, Phys. Rev. B 65, 081301 (2002)

[21] A. Wójs, I. Schlufarska, K.-S. Yi, J.J. Quinn, Phys. Rev. B 60, 11273 (1999); A. Wójs, P. Hawrylak, J.J. Quinn, 60, 11661 (1999).

[22] A. Wójs, J.J. Quinn, P. Hawrylak, Phys. Rev. B 62, 4630 (2000).

[23] A. Wójs, J.J. Quinn, Phys. Rev. B 63, 045303 (2001).

[24] A. Wójs, J.J. Quinn, Phys. Rev. B 63, 045304 (2001).

[25] A. Wójs, J.J. Quinn, Phys. Rev. B 66, 045323 (2002).

[26] J.J. Quinn, A. Wójs, in: 8th Intern. Workshop on Similarity in Diversity, University at Buffalo, SUNY Amherst (USA), May 15-17, 2002, Eds. D.L. Morabito, Y. Okamura, Nova 2003.

[27] T. Vanhoucke, M. Hayne, M. Henini, V.V. Moshchalkov, Phys. Rev. B 65, 233305 (2002).

[28] A. Wójs, J.J. Quinn, Philos. Mag. B 80, 1405 (2000).

[29] X.M. Chen, J.J. Quinn, Phys. Rev. Lett. 70, 2130 (1993).

[30] I. Szlufarska, A. Wójs, J.J. Quinn, Phys. Rev. B 64, 165318 (2001).

[31] C. Schüller, K.-B. Broocks, P. Schröter, Ch. Heyn, D. Heitmann, M. Bichler, W. Wegscheider, Tapash Chakraborty, V.M. Apalkov, Phys. Rev. Lett. 91, 116403 (2003).

[32] I. Szlufarska, A. Wójs, J.J. Quinn, Phys. Rev. B 66, 165318 (2001).

[33] N.R. Cooper, D.B. Chklovskii, Phys. Rev. B 55, 2436 (1997). 